\title{
Mass Transfer between Gas and Liquid in Bottom Blown Process*
}

\author{
By A. K. SINHA $A^{* *}$ and A. K. LAHIRI***
}

\begin{abstract}
Synopsis
Desorption of carbon dioxide from water during bottom blowing of nitrogen was studied. Volumetric mass-transfer coefficient of carbon dioxide was evaluated by following the change of carbon dioxide concentration in the bath. The volumetric mass-transfer coefficient was found to increase with the increase in the flow rate of nitrogen gas.

$A$ model for the estimation of volumetric mass-transfer coefficient was developed. The model uses Higbie equation for the estimation of masstransfer coefficient. The prediction of the model is in reasonable agreement with the experimental observations.
\end{abstract}

Key words: mass transfer; bottom blown process; kinetics; gas-liquid system.

\section{Introduction}

In the bottom blown processes, the gas jet coming out of the nozzles or porous plug forms large bubbles at their tip. These bubbles while ascending through the turbulent recirculating liquid disintegrate due to fluid-dynamic instability and form bubble swarm. These lead to rapid exchange of mass between the gas and liquid bath.

Mass transfer from a single bubble in static liquid bath has been widely investigated. Higbie equation provides a fair estimate of mass-transfer coefficient in the liquid phase for this case. ${ }^{1)}$ In gas stirred system, measurement of mass-transfer coefficient is difficult. So mostly volumetric mass-transfer coefficient, i.e., the product of mass-transfer coefficient and the area of bubble-liquid interface per unit volume of bath, is measured. Bhavaraju ${ }^{2)}$ showed volumetric masstransfer coefficient can be reasonably estimated using Higbie equation provided appropriate choice of various parameters are made. Recent results of Bessho $^{3)}$ on the carbon dioxide absorption into water also support this view.

Recently Kawakami ${ }^{4}$ measured rate of nitrogen transfer during submerged gas injection. Kawakami studied the radial distribution of nitrogen bubble frequency at different height in liquid pig iron bath and the rate of nitrogen absorption at $1250^{\circ} \mathrm{C}$. From their results they could obtain the value of masstransfer coefficient of nitrogen both in liquid and gas phase. Their results indicate that the mass-transfer coefficient in liquid phase for bubble stirred system is 5 to 9 times smaller than that in levitation or induction stirred melts.

In the present work, rate of carbon dioxide removal from the water bath during bottom blowing of nitrogen has been studied.

\section{Experimental}

The experimental setup was made up of a perspex cylindrical reaction vessel of height $0.45 \mathrm{~m}$ and diameter $0.3 \mathrm{~m}$. The gas was injected through a nozzle of $2 \mathrm{~mm}$ ID fitted at the centre of the base plate of reaction vessel. Most of the experiments were carried out with water bath height of $0.3 \mathrm{~m}$ and gas flow rates in the range of $3.33 \times 10^{-5} \sim 4.5 \times$ $10^{-4} \mathrm{Nm}^{3} / \mathrm{s}$.

Initially carbon dioxide was dissolved in the bath to near saturation level. Then nitrogen gas was passed through the nozzle at constant flow rate. Bath samples were collected at regular intervals to analyse the carbon dioxide content of the bath. Simultaneously two samples were collected from two different locations of the bath and the average carbon dioxide content of these two samples were taken as that of the bath.

\section{Results}

Mixing time studies for the system showed that the mixing time is in the range of $15 \sim 30 \mathrm{~s}$. The lower value corresponds to gas flow rate of $4.5 \times$ $10^{-4} \mathrm{~m} / \mathrm{s}$. This suggtests that at any instant the bath is not expected to be completely homogenious. However, assuming mixing time to be zero, the change in the carbon dioxide content of bath during nitrogen flushing is given by

$$
-\frac{d c}{d t}=K a\left(c-c_{e}\right)
$$

where, $c$ : the carbon dioxide concentration in the bath at any instant

$K$ : the mass-transfer coefficient of carbon dioxide

$a$ : the bubble-liquid interface area per unit volume of the bath

$c_{e}$ : the concentration of carbon dioxide in water which is in equilibrium with carbon dioxide in the nitrogen bubble.

Obviously, in the present case $c_{e} \simeq 0$ hence Eq. (1) becomes

$$
-\frac{d c}{d t}=K a c
$$

Integrating Eq. (2)

* Manuscript received on January 19, 1987; accepted in the final form on March 13, 1987. (C) 1987 ISIJ

** Formerly Department of Metallurgy, Indian Institute of Science, Bangalore, 560 012, India. Now at Department of Metallurgical Engineering, Ohio State University, Colombus, OH 43210, U.S.A.

*** Department of Metallurgy, Indian Institue of Science, Bangalore, 560 012, India. 


$$
-\ln \frac{c}{c_{0}}=K a t
$$

where, $c_{0}$ : the initial carbon dioxide concentration in the bath.

Figure 1 shows that experimental results for different flow rates. Inspite of scatter of the data points, it is apparent that the experimental results follow Eq. (3). Volumetric mass-transfer coefficient $K a$ is given by the slope of the straight lines.

Figure 2 shows that volumetric mass-transfer coefficient, $K a$, increases with the increase in gas flow rate. The effect of bath height on $K a$ in the range investigated does not appear to be significant.

\section{Theoretical Analysis and Discussion}

Figure 3 shows the idealised liquid circulation pattern in the bath. Bubbles in the central core move up and they drag liquid along with them as recirculating wakes. ${ }^{5)}$ This core is termed as plume zone. Outside this core the liquid flows downward.

Let $U_{G}$ be the velocity of the gas bubbles in the plume zone. Then the gas hold up in the bath, $v$, is given by

$$
v=\frac{Q H}{U_{G}}
$$

where, $Q$ : the flow rate of gas

$H$ : the bath height.

The bubble-liquid interfacial area for spherical bubbles in the plume zone is given by

$$
A=\frac{6 v}{d}
$$

where, $d$ : the mean diameter of the bubbles. In case of non-spherical bubbles, $d$ is taken as the diameter of volume equivalent sphere. Obviously in that case Eq. (5) is only an approximate one.

From Eqs. (4) and (5), gas-liquid interface area per unit volume of the bath is
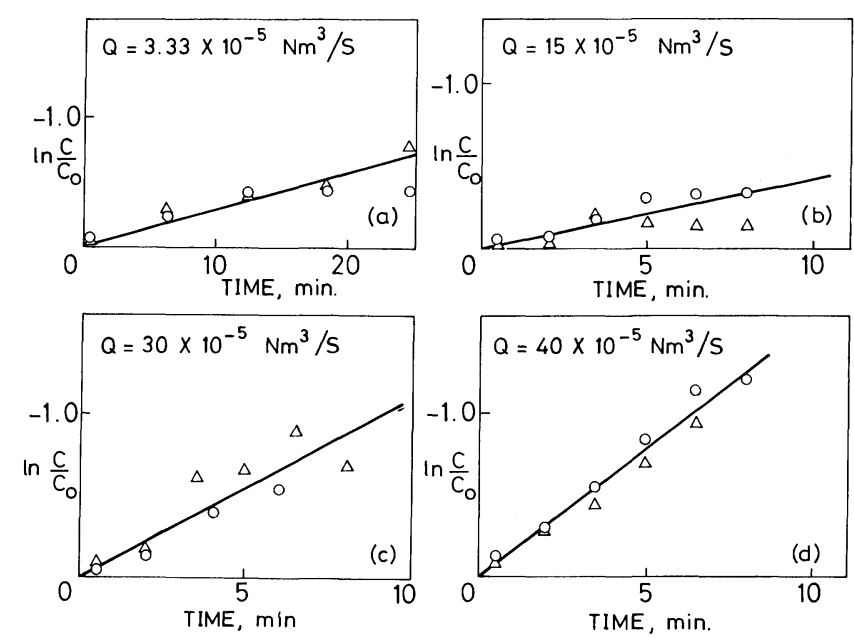

Experiment number 1

$\triangle$ Experiment number 2

Fig. 1. Variation of bath concentration of carbon dioxide with time for different flow rates.

$$
a=\frac{24 Q}{\Pi D_{b}^{2} U_{G} d}
$$

where, $D_{b}$ : the diameter of the bath.

Mass-transfer coefficient $K$ of any species according to Higbie equation is

$$
\kappa=\left(\frac{4 D U_{T}}{\Pi d}\right)^{1 / 2}
$$

where, $D:$ the diffusivity of the species in the liquid

$U_{T}$ : the terminal velocity of the bubble.

In the present case $U_{T}$ may be assumed as the slip velocity of the bubbles in the plume zone, i.e.,

$$
U_{G}=U_{L}+U_{T}
$$

where, $U_{L}$ : the velocity of the liquid in the plume zone.

When the diameter of the bubbles $d>0.0013 \mathrm{~m}, U_{T}$ may be approximately estimated by ${ }^{1)}$

$$
U_{T}=(2.14 \sigma / \rho d+0.505 g d)^{1 / 2}
$$

where, $\sigma, \rho$ : interfacial tension and density of the liquid, respectively.

Sano and Mori ${ }^{6)}$ showed that when fractional gas hold up in the plume zone is less than about 0.3 and diameter of plume zone is also less than half of the bath diameter

$$
U_{L}=1.17\left(Q_{g} H / A_{p}\right)^{0.346}
$$

where, $A_{p}$ : the area of the plume zone.

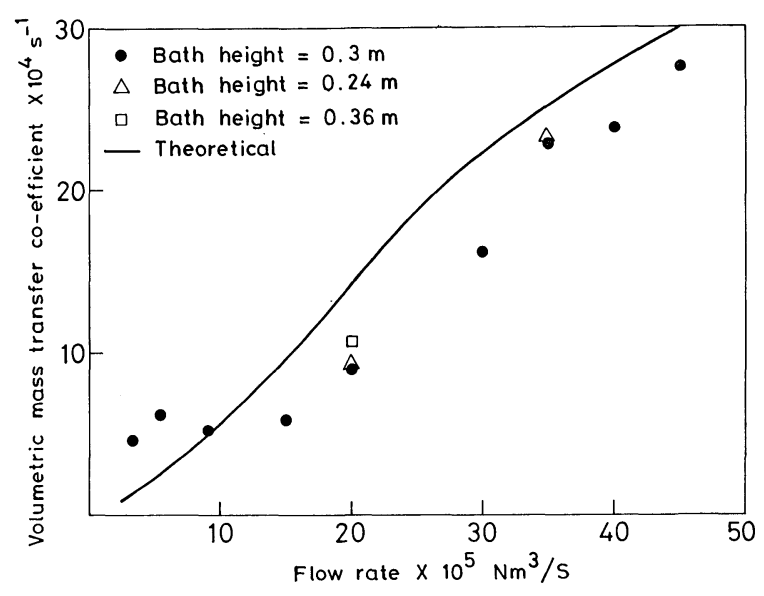

Fig. 2. Effect of gas flow rate on the volumetric masstransfer coefficient.

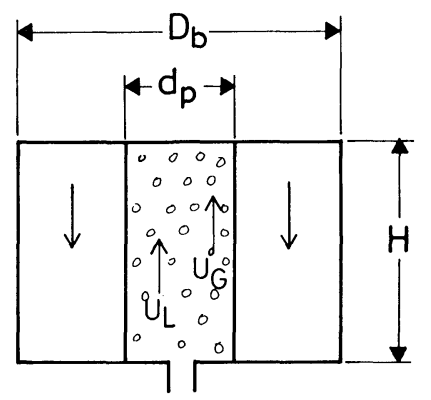

Fig. 3. Liquid circulation model. 
For the present experimental condition where the liquid flow in the plume zone was highly turbulent, (Reynolds number of the liquid in the plume zone was about 24000 for the lowest gas flow rate), bubble diameter is given $b^{2)}$

$$
\begin{array}{r}
d=0.7 \frac{\sigma^{0.6}}{p^{0.4} \rho^{0.2}}\left(\frac{\mu}{\mu_{g}}\right)^{0.1} \\
p=\frac{Q \rho_{g}}{A_{p}} \ldots \ldots \ldots
\end{array}
$$

where, $\mu_{g}, \mu$ : the viscosity of the gas and liquid, respectively.

When $d$ calculated by Eq. (11) is less than $0.0045 \mathrm{~m}$, diameter of the bubble is taken as ${ }^{2)}$

$$
d=0.0045 \mathrm{~m}
$$

The estimation of volumetric mass-transfer coefficient, $K a$, by Eqs. (6) (13), requires the knowledge of plume diameter. Assuming that the plume diameter, $d_{p}$, is same as the gas jet diameter on the top surface of the bath and bath depth is much greater than the nozzle diameter;

$$
d_{p}=2 H \tan \frac{\theta_{c}}{2}
$$

where, $\theta_{c}$ : the jet cone angle.

Themelis ${ }^{7)}$ found that $\theta_{c}$ for air-water system is about $20^{\circ}$ hence Eq. (14) becomes

$$
d_{p}=0.35 H
$$

In the present case, plume diameter was estimated by visual observation as $0.08 \mathrm{~m}$ for $0.3 \mathrm{~m}$ bath height. This figure compares favourably with the estimate obtained from Eq. (15).

Figure 2 shows the calculated values of $K a$ for bath height of $0.3 \mathrm{~m}$ along with the experimental results. Diffusivity of carbon dioxide in water was taken $^{8)}$ as $D=1.8 \times 10^{-9} \mathrm{~m}^{2} \mathrm{~s}^{-1}$. It is apparent from the figure that calculated values give a rough estimation of volumetric mass-transfer coefficient. At low flow rates, orifice Reynolds number less than 5000 , calculated values are less than the experimental ones but at higher flow rates the calculated values are greater than the experimental ones and the difference between the two seems to decrease with the increase in flow rates. Equations (6) (13) indicate that $K a$ is roughly proportional to $d^{-1.5}$ and independent of bath height. Thereby the estimation of $K a$ by the above mentioned equations is very sensitive to the estimation of the diameter of the bubbles.

Kawakami $^{4)}$ reported $K=1.05 \times 10^{-5} \mathrm{~m} / \mathrm{s}$ and $K a$ $=3.9 \times 10^{-4} \mathrm{~s}^{-1}$ for transfer of nitrogen in liquid pig iron at $1250^{\circ} \mathrm{C}$ when nitrogen was injected at a flow rate of $0.33 \times 10^{-3} \mathrm{Nm}^{3} / \mathrm{s}$. Diffusivity of nitrogen in liquid pig iron is not known. Extrapolation of high temperature data ${ }^{9)}$ of diffusivity of nitrogen in pure iron show that at $1250^{\circ} \mathrm{C}$ diffusivity is equal to $3.12 \times 10^{-9} \mathrm{~m}^{2} / \mathrm{s}$. Sano and Mori ${ }^{10}$ ) showed that the bubble diameter in liquid metal may be estimated by

$$
d=6.83\left(\frac{\sigma}{\rho}\right)^{0.5} V_{s}^{0.44}
$$

where, $V_{s}$ : the superficial velocity of the injected gas in the bath.

Calculation shows that for the experimental condition of Kawakami, $K=2.2 \times 10^{-4} \mathrm{~m} / \mathrm{s}$ and $K a=7.5 \times$ $10^{-4} \mathrm{~s}^{-1}$. It may be noted that although the calculated value of mass-transfer coefficient is about 20 times larger than the experimental ones, the agreement for $K a$ is reasonable. This observation and present experimental results suggest that although Higbie model, Eq. (7), may not be valid for the submerged gas injection system, an estimate of volumetric mass-transfer coefficient can be obtained using the same.

\section{Conclusion}

(i) Volumetric mass-transfer coefficient of carbon dioxide in water increases with the increase in the flow rate of injected nitrogen.

(ii) A reasonable estimate of the volumetric masstransfer coefficient can be obtained using Higbie equation for mass-transfer coefficient.

(iii) Estimation of mass-transfer coefficient by Higbie equation may be wrong by order of magnitude.

\section{REFERENGES}

1) R. Clift, J. R. Grace and M. E. Weber: Bubbles, Drops and Particles, Academic Press, New York, (1978), 169.

2) S. M. Bhavaraju, T.W.F. Russel and H.W. Blanch: AIChE J., 24 (1978), 454.

3) N. Bessho, S. Taniguchi and A. Kikuchi: Tetsu-to-Hagané, 71 (1985), 1623.

4) M. Kawakami, Y. Kitazawa, T. Nakamura, T. Miyake and K. Ito, Trans. Iron Steel Inst. Jpn., 25 (1985), 394.

5) Y. Sahai and R.I.L. Guthrie: Metall. Trans. B, 13B (1982), 193.

6) M. Sano and K. Mori: Trans. Iron Steel Inst. Jpn., 23 (1983), 169.

7) N. J. Themelis, P. Tarasoff and J. Szekely: Trans. Metall. Soc. AIME, 245 (1969), 2425.

8) D. M. Himmelblau: Chem. Rev., 64 (1964), 527.

9) M. Inouye, Y. Kojima, T. Choh, S. Uekawa and Y. Yamada: Trans. Iron Steel Inst. Jpn., 13 (1973), 29.

10) M. Sano and K. Mori: Trans. Iron Steel Inst. Jpn., 20 (1980), 675 . 